\title{
Plasma glial fibrillary acidic protein is elevated in cognitively normal older adults at risk of Alzheimer's disease
}

Pratishtha Chatterjee (1) ${ }^{1,2}$, Steve Pedrini ${ }^{2}$, Erik Stoops ${ }^{3}$, Kathryn Goozee $\mathbb{1}^{1,24,5,6,7}$, Victor L. Villemagne ${ }^{8}$, Prita R. Asih', Inge M. W. Verberk (1) ${ }^{9}$, Preeti Dave ${ }^{1,5}$, Kevin Taddei ${ }^{2,10}$, Hamid R. Sohrabi ${ }^{12,10,11}$, Henrik Zetterberg (1) $2,13,14,15$,

Kaj Blennow (1) ${ }^{12,13}$, Charlotte E. Teunissen ${ }^{9}$, Hugo M. Vanderstichele ${ }^{16}$ and Ralph N. Martins (10 ${ }^{1,2,4,6,7,10}$

\begin{abstract}
Glial fibrillary acidic protein (GFAP), an astrocytic cytoskeletal protein, can be measured in blood samples, and has been associated with Alzheimer's disease (AD). However, plasma GFAP has not been investigated in cognitively normal older adults at risk of $A D$, based on brain amyloid- $\beta(A B)$ load. Cross-sectional analyses were carried out for plasma GFAP and plasma $A \beta 1-42 / A \beta 1-40$ ratio, a blood-based marker associated with brain $A \beta$ load, in participants (65-90 years) categorised into low $(A \beta-, n=63)$ and high $(A \beta+, n=33)$ brain $A \beta$ load groups via $A \beta$ positron emission tomography. Plasma GFAP, $A \beta 1-42$, and $A \beta 1-40$ were measured using the Single molecule array (Simoa) platform. Plasma GFAP levels were significantly higher $(p<0.00001)$, and plasma $A \beta 1-42 / A \beta 1-40$ ratios were significantly lower $(p<0.005)$, in $A \beta+$ participants compared to $A \beta$ - participants, adjusted for covariates age, sex, and apolipoprotein $E$ $\varepsilon 4$ carriage. A receiver operating characteristic curve based on a logistic regression of the same covariates, the base model, distinguished $A \beta+$ from $A \beta$ - (area under the curve, $A U C=0.78$ ), but was outperformed when plasma GFAP was added to the base model ( $A \cup C=0.91)$ and further improved with plasma $A \beta 1-42 / A \beta 1-40$ ratio $(A \cup C=0.92)$. The current findings demonstrate that plasma GFAP levels are elevated in cognitively normal older adults at risk of AD. These observations suggest that astrocytic damage or activation begins from the pre-symptomatic stage of AD and is associated with brain A $\beta$ load. Observations from the present study highlight the potential of plasma GFAP to contribute to a diagnostic blood biomarker panel (along with plasma A $\beta 1-42 / A \beta 1-40$ ratios) for cognitively normal older adults at risk of AD.
\end{abstract}

\section{Introduction}

Alzheimer's disease (AD) is the most common form of dementia and it is estimated that globally over 50 million people are living with $\mathrm{AD}$ or other forms of dementia ${ }^{1}$. Currently, there is no cure or effective treatment for $\mathrm{AD}$ despite all scientific efforts and therefore, more recent clinical trials are focussing on prevention programmes for

Correspondence: Ralph N. Martins (r.martins@ecu.edu.au)

'Department of Biomedical Sciences, Macquarie University, North Ryde, NSW, Australia

${ }^{2}$ School of Medical and Health Sciences, Edith Cowan University, Joondalup, WA, Australia

Full list of author information is available at the end of the article
$\mathrm{AD}$, thereby requiring the identification of populations at risk of $\mathrm{AD}$.

Extracellular amyloid- $\beta(A \beta)$ plaques and intracellular neurofibrillary tangles comprising tau are the major neuropathological hallmarks of $\mathrm{AD}$ and while a postmortem examination identifying these hallmarks is relied upon for a confirmative diagnosis, $A \beta$ and tau neuropathology associated with $\mathrm{AD}$ can be identified in vivo via positron emission tomography (PET) and cerebrospinal fluid (CSF) analysis 15-20 years prior to symptom onset ${ }^{2}$. However, the cost of PET imaging, the throughput of imaging in general and the invasiveness of lumbar puncture, required for CSF sample collection, restrict the

\section{(c) The Author(s) 2021}

(c) Open Access This article is licensed under a Creative Commons Attribution 4.0 International License, which permits use, sharing, adaptation, distribution and reproduction cc) in any medium or format, as long as you give appropriate credit to the original author(s) and the source, provide a link to the Creative Commons license, and indicate if changes were made. The images or other third party material in this article are included in the article's Creative Commons license, unless indicated otherwise in a credit line to the material. If material is not included in the article's Creative Commons license and your intended use is not permitted by statutory regulation or exceeds the permitted use, you will need to obtain permission directly from the copyright holder. To view a copy of this license, visit http://creativecommons.org/licenses/by/4.0/. 
implementation of these markers in standard clinical practice and as screening tools in clinical trials. In contrast, the cost effective and less invasive nature of bloodbased biomarkers could serve as attractive surrogate markers for initial clinical diagnostic testing and screening for clinical trials.

Glial fibrillary acidic protein (GFAP) is an astrocytic cytoskeletal protein that serves as a marker of abnormal activation and proliferation of astrocytes due to neuronal damage, also known as astrogliosis ${ }^{3}$. Astrogliosis has also been observed around $A \beta$ plaques from the prodromal stages of $\mathrm{AD}$, such as the mild cognitive impairment stage $^{4}$, and GFAP expression has been reported to correlate with $A \beta$ plaque density in $\mathrm{AD}$ brain tissue ${ }^{5}$. In addition, higher GFAP levels have been reported in CSF samples from individuals with $\mathrm{AD}$ and other dementias, compared to healthy controls ${ }^{6}$.

Interestingly, relatively recent studies have also reported higher GFAP levels in the blood in early and late-onset $\mathrm{AD}^{7-9}$. GFAP levels in the blood were also observed to inversely correlate with cognition ${ }^{8}$ and positively correlate with the extent of white matter injury ${ }^{7}$. The current study investigated whether elevated GFAP levels in the blood precede the onset of the clinical symptoms of $\mathrm{AD}$ in cognitively normal older adults at risk of AD.

Given that the onset of abnormal brain $A \beta$ load buildup assessed using PET begins as early as two decades prior to the clinical manifestation of $\mathrm{AD}$, and is a prodromal feature and biomarker of $\mathrm{AD}^{2,10}$, plasma GFAP levels were compared between cognitively normal older adults with low brain $A \beta$ load $(A \beta-)$ and cognitively normal older adults at risk of $A D$, due to high brain $A \beta$ load, $(\mathrm{A} \beta+)^{11}$. Our hypothesis was that plasma GFAP levels will be higher in the $A \beta+$ group compared to the $\mathrm{A} \beta$ - group.

The current study also evaluated the potential of plasma GFAP in differentiating between $A \beta+$ and $A \beta$ - individuals. In addition, given that the association between plasma $A \beta 42 / A \beta 40$ ratios and brain $A \beta$ load has been extensively reported ${ }^{12-15}$, this study also evaluated the combined potential of plasma GFAP and plasma $A \beta 1-42$ / $A \beta 1-40$ ratios in discriminating between $A \beta+$ and $A \beta-$ individuals. Furthermore, associations of plasma GFAP with the $\mathrm{AD}$ risk factors, cognitive performance, and neurodegeneration marker, neurofilament light (NF-L), were also assessed in the present study.

\section{Materials and methods}

\section{Participants and cognitive assessments}

Participants in the current study were from the Kerr Anglican Retirement Village Initiative in Aging Health (KARVIAH) cohort $^{16}$. All participants from the KARVIAH cohort met the inclusion and exclusion criteria, wherein the inclusion criteria comprised an age range of 65-90 years, good general health, no known significant cerebral vascular disease, fluent in English, adequate/corrected vision and hearing to enable testing, and no dementia or other pathological cognitive impairment, as primarily screened by a Montreal Cognitive Assessment (MoCA) score $\geq 26$. MoCA scores lying between 18 and 25 were assessed on a case by case basis by the study neuropsychologist following stratification of scores, using age and education-adjusted norms ${ }^{17}$. The exclusion criteria comprised, previous diagnosis of dementia ${ }^{18}$, presence of acute functional psychiatric disorder (including lifetime history of schizophrenia or bipolar disorder), history of stroke, severe or extremely severe depression (based on the Depression Anxiety Stress Scales; DASS), and uncontrolled hypertension (systolic $\mathrm{BP}>170 \mathrm{~mm} \mathrm{Hg}$ or diastolic $\mathrm{BP}>100 \mathrm{~mm} \mathrm{Hg}$ ). From the volunteers who met the inclusion and exclusion criteria $(n=134), 105$ participants underwent neuroimaging, neuropsychometric evaluation, and blood collection since the remaining participants declined undergoing neuroimaging or withdrew from the study. Within these 105 participants, 100 participants were considered to have normal global cognition based on their Mini-Mental State Examination (MMSE; scores can range from 0 to 30, with higher scores indicating better cognitive function $)^{19}$ wherein, a cut-off score $<26$ was employed to screen out potential dementia patients. Plasma GFAP concentrations were measured in 96 of the 100 participants, and plasma $A \beta 1-40$ and $A \beta 1-42$ concentrations were measured in 95 of these 100 participants. However, the total set of GFAP, A $\beta 1-40$, and A $\beta 1-42$ concentrations were available in 94 of these participants. In addition, participants with a Memory Assessment Clinic-Questionnaire (MAC-Q) score of 25-35 were considered as subjective memory complainers (SMCs, $n=$ 74; a specific form of subjective cognitive decline, defined by self-reported memory complaints), while those with a MAC-Q score $\leq 24$ were considered as non-complainers $(n=22)$. Details of the participants included within the current study have been illustrated in Supplementary Fig. 1 .

Further, cognitive measures were calculated for verbal and visual episodic memory, working memory and executive function, as well as for a global composite score, that included verbal and visual episodic memory, working memory and executive function and MMSE scores, for each participant as described previously ${ }^{20}$.

All volunteers provided written informed consent prior to participation, and the Bellberry Human Research Ethics Committee, Australia (reference number 2012-09-1086) and the Macquarie University Human Research Ethics Committee (reference number 5201701078) provided approval for the study.

\section{Evaluation of neocortical amyloid- $\beta$ load via PET}

All study participants were imaged within 3 months of blood collection wherein participants underwent $A \beta$ PET 
imaging with ${ }^{18} \mathrm{~F}$-florbetaben (FBB) at Macquarie Medical Imaging in Sydney, Australia. Participants were administered an intravenous bolus of FBB slowly over $30 \mathrm{~s}$, while in a rested position. Images were acquired over a $20 \mathrm{~min}$ scan, in $5 \mathrm{~min}$ acquisitions, beginning $50 \mathrm{~min}$ post injection. Brain $\mathrm{A} \beta$ load was calculated, using $\mathrm{CapAIBL}^{21}$, as the mean standard uptake value ratio (SUVR) of the neocortical region, including the frontal, superior parietal, lateral temporal, lateral occipital, and anterior and posterior cingulate regions normalised to the cerebellar cortex. A cut-off score of 1.35 SUVR was used to categorise participants with low brain $A \beta$ load $(A \beta-$, SUVR < 1.35) and high brain $A \beta$ load $(A \beta+, \text { SUVR } \geq 1.35)^{16}$.

\section{Blood collection, measurement of plasma GFAP, plasma $A \beta$ and NF-L, and APOE genotyping}

All study participants fasted for a minimum of $10 \mathrm{~h}$ overnight prior to blood withdraw employing standard serological methods and processing ${ }^{16}$. Following blood sample processing, plasma fractions were stored at $-80^{\circ} \mathrm{C}$ until further testing ${ }^{16}$. Plasma GFAP concentrations were measured at Amsterdam University Medical Centers using the Simoa ${ }^{\mathrm{TM}}$ GFAP Discovery Kit on the ultrasensitive Single molecule array (Simoa) platform (HDx instrument) according to the manufacturer's instructions (catalogue number 102336, Quanterix, Massachusetts, USA). Briefly, plasma samples were added into the aspiration plate of the instrument and diluted four times using assay diluent. Samples were then incubated simultaneously with the capture beads and biotinylated conjugate for $35 \mathrm{~min} 15 \mathrm{~s}$ followed by a wash step and incubation of streptavidin-ß-galactosidase (SBG) for $5 \mathrm{~min} 15 \mathrm{~s}$. Following a next wash step, the beads were resuspended in a resorufin B-D-galactopyranoside (RGP) substrate solution for signal generation. GFAP concentrations were calculated using a 4PL 1/Y2 weighted curve fit on the basis of seven calibrator points (excluding the blank value) between 1.37 and $1000 \mathrm{pg} / \mathrm{mL}$, according to the manufacturer's instructions. The calibrator points were prepared by serial dilution using a stock of concentrated calibrator included in the test kit. Three serum pools spiked with CSF, to obtain three levels (high-medium-low), served as QC samples with average GFAP concentrations of respectively 283.0, 61.0, and $13.6 \mathrm{pg} / \mathrm{mL}$. The repeatability and reproducibility over the three control samples over the two test runs ranged respectively between $0-14(\% \mathrm{CV})$ and $8-16(\% \mathrm{CV})$.

Plasma $A \beta$ concentrations were measured employing the Amyblood test that was developed at Amsterdam University Medical Centers in collaboration with ADx NeuroSciences (Ghent, Belgium), on the Simoa platform (HDx instrument, Quanterix), using monoclonal antibodies provided by ADx NeuroSciences ${ }^{22}$. For A $\beta 1-40$, C-terminal-specific $\mathrm{ADx} 103(2 \mathrm{G} 3, \mathrm{~A} \beta \mathrm{x}-40)$ was used as the capture antibody and N-terminal specific ADx101 $(3 D 6, A \beta 1-x)$ was used as the detector antibody ${ }^{23}$. For A $\beta 1-42, C$-terminal-specific ADx102 (21F12, $x-42)$ was used as the capture antibody and N-terminal-specific ADx101 (3D6) was used as the detector antibody ${ }^{23}$. Briefly, plasma samples were prediluted 20 times for $\mathrm{A} \beta 1-40$ and four times for $\mathrm{A} \beta 1-42$ into a 96-well polypropylene pre-dilution plate, using assay diluent. Samples were incubated simultaneously with the capture beads and biotinylated conjugate for $60 \mathrm{~min}$ followed by a wash step and incubation of SBG for $5 \mathrm{~min} 15 \mathrm{~s}$. Following a next wash step, the beads were resuspended in a RGP substrate solution for signal generation. For both $A \beta 1-40$ and $A \beta 1-42$, seven non-blank calibrator points in ready to use format (in assay diluent) were used between 1 and $20 \mathrm{pg} / \mathrm{mL}$. The analyte concentrations were calculated using a 4PL non-weighted curve fit. Three non-spiked individual EDTA plasma samples served as QC samples for both $A \beta 1-40$ and $A \beta 1-42$ assays, with an average concentration of respectively $60.7,112.8$, and $74.4 \mathrm{pg} / \mathrm{mL}$ for $\mathrm{A} \beta 1-40$, and $16.9,24.7$, and $18.5 \mathrm{pg} / \mathrm{mL}$ for $\mathrm{A} \beta 1-42$. The repeatability over the three control samples over all duplicate values ranged between $0-4(\% \mathrm{CV})$ and $0-19$ (\% $C V)$ for $A \beta 1-40$ and $A \beta 1-42$, respectively. The reproducibility over the two independent test runs ranged between 3-7 (\%CV) and $0-16(\% \mathrm{CV})$ for $\mathrm{A} \beta 1-40$ and A $\beta 1-42$, respectively.

Plasma NF-L concentrations were also measured using the Simoa platform, as described previously ${ }^{24}$, using two non-competing monoclonal antibodies wherein capture antibody 47:3 and detector antibody 2:1 were used (Uman Diagnostics, Sweden). Apolipoprotein $\mathrm{E}(A P O E)$ genotype was determined from purified genomic DNA extracted from $0.5 \mathrm{~mL}$ whole blood, as previously described ${ }^{16}$.

\section{Statistical analyses}

Descriptive statistics including means and standard deviations were calculated for $\mathrm{A} \beta-$ and $\mathrm{A} \beta+$ groups, with comparisons employing Student's $t$ tests or Chi-square tests as appropriate. Linear models were employed to compare continuous variables between $\mathrm{A} \beta-$ and $\mathrm{A} \beta+$ groups corrected for covariates age, sex, and $A P O E \varepsilon 4$ carrier status. Dependent variables were natural $\log$ transformed to better approximate normality and variance homogeneity as required. Spearman's correlation coefficient $\left(r_{\mathrm{s}}\right)$ was employed to investigate correlations between continuous parameters. Logistic regression with $\mathrm{A} \beta-/+$ as response was used to evaluate predictive models and receiver operating characteristic (ROC) curves constructed from the logistic scores. All analyses were carried out using IBM ${ }^{\circledR}$ SPSS $^{\circledR}$ Version 23 and ROC curves were generated using the package Deducer on $\mathrm{R}$ (version 3.2.5). 


\section{Results}

\section{Cohort characteristics}

Study participant characteristics are presented in Table 1. No significant differences were observed in sex, age, body mass index (BMI), MMSE scores, and the number of SMCs between $A \beta-$ and $A \beta+$ participants. However, the $A P O E \varepsilon 4$ carriage frequency was significantly higher in the $\mathrm{A} \beta+$ group compared to the $\mathrm{A} \beta$ - group, as expected ${ }^{25}$ (Table 1).

\section{Comparison of plasma GFAP between $A \beta$ - participants and $A \beta+$ participants}

Plasma GFAP concentrations were significantly higher in the $\mathrm{A} \beta+$ group $(n=33)$ compared to the $\mathrm{A} \beta$ - group $(n=63)$, before and after adjusting for potential risk factors, age, sex, and $A P O E \& 4$ status (Fig. 1 and Table 2, $p<0.0001)$.

On stratifying study participants based on subjective memory complaints (SMC: $n=74$ and non-SMC: $n=22$ ), plasma GFAP continued to remain significantly higher in the A $\beta+\operatorname{SMCs}(n=25)$ compared to A $\beta-\operatorname{SMCs}(n=49)$ before and after adjusting for covariates age, sex, and $A P O E \& 4$ status (Table 2, $p<0.0001$ ). In the non-SMCs, plasma GFAP was observed to be significantly higher in $\mathrm{A} \beta+$ non-SMCs $(n=8)$ compared to the A $\beta$ - non-SMCs $(n=14)$ after adjusting for the aforementioned covariates (Table 2, $p<0.05$ ).

On stratifying study participants by $A P O E \varepsilon 4$ carriage ( $\varepsilon 4$ non-carriers: $n=77$ and $\varepsilon 4$ carriers: $n=19$ ), significantly higher plasma GFAP concentrations were observed in the $\mathrm{A} \beta+$ group $(n=19)$ compared to the $\mathrm{A} \beta-$ group $(n=58)$ within the $A P O E \& 4$ non-carriers, before and after adjusting for potential risk factors, age, and sex (Supplementary Table 1, $p<0.0001$ ). Within the

Table 1 Cohort characteristics.

\begin{tabular}{llll}
\hline & A $\boldsymbol{\beta}-$ & $\mathbf{A} \boldsymbol{\beta}+$ & $\boldsymbol{p}$ \\
\hline Sex (male/female) & $18 / 45$ & $13 / 20$ & 0.281 \\
Age (years, mean \pm SD) & $77.41 \pm 5.45$ & $79.64 \pm 5.20$ & 0.057 \\
BMI (mean \pm SD) & $27.28 \pm 4.51$ & $27.36 \pm 3.79$ & 0.927 \\
APOE E4 carriers (N (\%)) & $5(7.94)$ & $14(42.42)$ & $<0.0001$ \\
MMSE (mean \pm SD) & $28.52 \pm 1.16$ & $28.82 \pm 1.07$ & 0.230 \\
Subjective memory complainers & $49(77.8)$ & $25(75.76)$ & 0.823 \\
(N (\%)) & & & \\
FBB-PET SUVR (mean \pm SD) & $1.16 \pm 0.09$ & $1.71 \pm 0.26$ & - \\
\hline
\end{tabular}

Baseline characteristics including sex, age, body mass index (BMI), APOE $\varepsilon 4$ status, Mini-mental State Examination (MMSE) scores, subjective memory complainer status (assessed by the Memory Assessment Clinic-Questionnaire (MAC-Q) score), and brain $A \beta$ load represented by the standard uptake value ratio (SUVR) of ligand ${ }^{18} \mathrm{~F}$-florbetaben (FBB) in the neocortical region normalised with that in the cerebellum, have been compared between $A \beta-$ (SUVR $<1.35, n$ $=63)$ and $A \beta+(S U V R \geq 1.35, n=33)$ study participants. Chi-square tests or linear models were employed as appropriate.
$A P O E \& 4$ carriers, no significant difference in GFAP concentration was observed between the $\mathrm{A} \beta+$ group $(n=$ 14) compared to the $A \beta-$ group $(n=5$; Supplementary Table 1). This observation could be attributed to the modest sample size of the $\varepsilon 4$ carrier subset available within the current study.

\section{Comparison of plasma $A \beta 1-40, A \beta 1-42$, and $A \beta 1-42$ / $A \beta 1-40$ ratios between $A \beta$ - participants and $A \beta+$ participants}

Plasma $A \beta 1-40$ and $A \beta 1-42$ concentrations and plasma $\mathrm{A} \beta 1-42 / \mathrm{A} \beta 1-40$ ratios, measured in the study participants have been presented in Table 3. While no significant differences were observed in plasma $A \beta 1-40$ concentrations between the $\mathrm{A} \beta-(n=62)$ and $\mathrm{A} \beta+$ groups $(n=33)$, significant differences in plasma $A \beta 1-42$ concentrations and $A \beta 1-42 / A \beta 1-40$ ratios were observed between the two groups, wherein plasma $A \beta 1-42$ concentrations and $A \beta 1-42 / A \beta 1-40$ ratios were lower in the $\mathrm{A} \beta+$ group compared to the $\mathrm{A} \beta$ - group before and after correcting for covariates age, sex, and $A P O E \& 4$ status (Fig. 1 and Table 3, $p<0.05$ ).

On stratifying study participants based on subjective memory complaints (SMC: $n=75$ and non-SMC: $n=20$ ), plasma $A \beta 1-42$ concentrations, and $A \beta 1-42 / A \beta 1-40$ ratios continued to remain significantly lower in the $A \beta+$ SMCs $(n=25)$ compared to A $\beta-\operatorname{SMCs}(n=50)$ before and after correcting for covariates age, sex, and $A P O E$ $\varepsilon 4$ status (Table $3, p<0.05$ ). However, no significant difference was observed in plasma $A \beta 1-42$ concentrations, and $A \beta 1-42 / A \beta 1-40$ ratios between $A \beta+$ non-SMCs $(n=8)$ and $\mathrm{A} \beta-$ non-SMCs $(n=12$; Table 3$)$.

On stratifying study participants by $A P O E \varepsilon 4$ carriage ( $\varepsilon 4$ non-carriers: $n=76$ and $\varepsilon 4$ carriers: $n=19$ ), significantly lower plasma $A \beta 1-42$ concentrations and $\mathrm{A} \beta 1-42 / \mathrm{A} \beta 1-40$ ratios were observed in the $\mathrm{A} \beta+$ group $(n=19)$ compared to the $\mathrm{A} \beta-$ group $(n=57)$, within the $A P O E \varepsilon 4$ non-carrier group, after adjusting for potential risk factors, age, and sex (Supplementary Table 2, p < 0.05). Within the $A P O E \& 4$ carrier group, no significant differences in plasma A $\beta 1-42$ concentrations and $\mathrm{A} \beta 1-42 / \mathrm{A} \beta 1-40$ ratios were observed between the $\mathrm{A} \beta+$ group $(n=14)$ compared to the $\mathrm{A} \beta-$ group $(n=5$; Supplementary Table 2 ). This observation could be attributed to the modest sample size of the $\varepsilon 4$ carrier subset available within the current study.

\section{Evaluation of plasma GFAP and $A \beta 1-42 / A \beta 1-40$ ratios as predictors of brain $A \beta$ status}

Plasma GFAP and $A \beta 1-42 / A \beta 1-40$ ratios were evaluated as potential markers for differentiating between $A \beta+$ and $A \beta-$ participants, using logistic regression with $\mathrm{A} \beta+$ or $\mathrm{A} \beta-$ as response. A 'base' model incorporating the major risk factors for $\mathrm{AD}$, namely age, sex, and $A P O E$ 

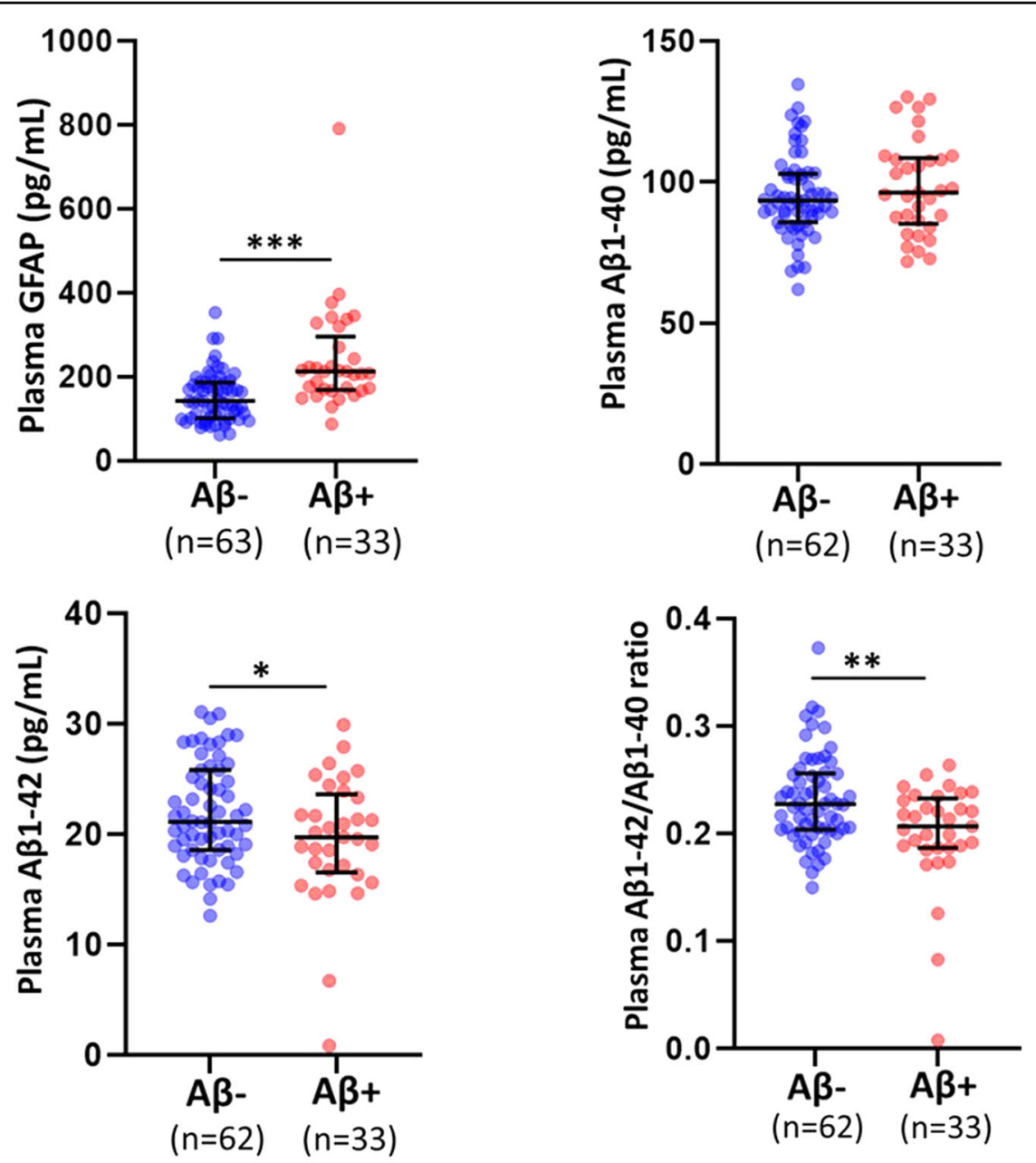

Fig. 1 Comparison of plasma GFAP, $A \beta 1-40, A \beta 1-42$, and $A \beta 1-42 / A \beta 1-40$ ratios between $A \beta-$ and $A \beta+$ cognitively normal older adults. Plasma GFAP, $A \beta 1-40$ and $A \beta 1-42$ levels, and plasma $A \beta 1-42 / A \beta 1-40$ ratios were compared between cognitively normal older adults with low brain $A \beta$ load $(A \beta-)$ and high brain $A \beta$ load $(A \beta+)$ using linear models. Plasma GFAP concentrations were significantly higher, and plasma $A \beta 1-42 / A \beta 1-40$ ratios were significantly lower in $A \beta+$ participants compared to $A \beta$ - participants. The line segment within each jitter plot represents the median of the data and error bars in the graphs represent the interquartile range for the $A \beta$ - and $A \beta+$ groups. ${ }^{*} p<0.05,{ }^{* *} p \leq 0.001,{ }^{* * *} p \leq 0.0001$.

Table 2 Comparison of plasma GFAP between $A \beta-$ and $A \beta+$ participants.

\begin{tabular}{|c|c|c|c|c|c|c|}
\hline & $A \beta-$ & $(95 \% \mathrm{Cl})$ & $A \beta+$ & $(95 \% \mathrm{Cl})$ & $p$ & $p^{a}$ \\
\hline \multirow[t]{2}{*}{ All participants } & $n=63$ & & $n=33$ & & & \\
\hline & $151.42 \pm 58.49$ & (129.66-173.18) & $240.12 \pm 124.88$ & $(210.05-270.18)$ & $7 E-6$ & $5.76 \mathrm{E}-7$ \\
\hline \multirow[t]{2}{*}{ SMC } & $n=49$ & & $n=25$ & & & \\
\hline & $152.73 \pm 58.18$ & $(126.35-179.11)$ & $252.22 \pm 137.75$ & (215.29-289.16) & ${ }^{b} 9 E-6$ & $4.8 \mathrm{E}-5$ \\
\hline \multirow[t]{2}{*}{ Non-SMC } & $n=14$ & & $n=8$ & & & \\
\hline & $146.83 \pm 61.58$ & (112.06-181.60) & $202.28 \pm 63.81$ & $(156.28-248.27)$ & 0.059 & 0.020 \\
\hline
\end{tabular}

Plasma glial fibrillary acidic protein (GFAP) levels were compared between cognitively normal older adults with low brain $A \beta$ load $(A \beta-)$ and high brain $A \beta$ load ( $A \beta+)$ using linear models. All participants were further categorised into subjective memory complainers (SMC, $n=74)$ and non-complainers (non-SMC, $n=22)$. Data are presented in mean $\pm \mathrm{SD}$ in $\mathrm{pg} / \mathrm{mL}$. $p$ values in bold font were considered as significant $(p<0.05)$.

${ }^{a} p$ represents $p$ values adjusted for age, sex, and APOE $\varepsilon 4$ status.

${ }^{\mathrm{b}}$ Represents $p$ values obtained from natural log transformed GFAP concentrations to better approximate normality when required. 
Table 3 Comparison of plasma $A \beta 1-40, A \beta 1-42$, and $A \beta 1-42 / A \beta 1-40$ ratios between $A \beta$ - and $A \beta+$ participants.

\begin{tabular}{|c|c|c|c|c|c|c|}
\hline & $A \beta-$ & $(95 \% \mathrm{Cl})$ & $A \beta+$ & $(95 \% \mathrm{Cl})$ & $p$ & $p^{a}$ \\
\hline All participants & $n=62$ & & $n=33$ & & & \\
\hline$A \beta 1-40$ & $95.19 \pm 14.78$ & $(91.27-99.10)$ & $98.37 \pm 16.88$ & $(93.00-103.74)$ & 0.344 & 0.659 \\
\hline$A \beta 1-42$ & $21.96 \pm 4.58$ & $(20.70-23.22)$ & $19.54 \pm 5.74$ & $(17.81-21.27)$ & 0.027 & 0.022 \\
\hline$A \beta 1-42 / A \beta 1-40$ ratio & $0.232 \pm 0.042$ & $(0.221-0.243)$ & $0.200 \pm 0.050$ & $(0.184-0.215)$ & 0.001 & 0.004 \\
\hline SMC & $n=50$ & & $n=25$ & & & \\
\hline$A \beta 1-40$ & $95.62 \pm 14.74$ & (91.19-100.05) & $98.67 \pm 17.54$ & $(92.41-104.94)$ & 0.430 & 0.624 \\
\hline$A \beta 1-42$ & $22.05 \pm 4.69$ & (20.58-23.52) & $19.12 \pm 6.17$ & $(17.02-21.19)$ & 0.024 & 0.015 \\
\hline$A \beta 1-42 / A \beta 1-40$ ratio & $0.232 \pm 0.044$ & $(0.218-0.245)$ & $0.195 \pm 0.056$ & $(0.176-0.214)$ & 0.003 & 0.003 \\
\hline Non-SMC & $n=12$ & & $n=8$ & & & \\
\hline$A \beta 1-40$ & $93.39 \pm 15.48$ & $(83.96-102.83)$ & $97.41 \pm 15.68$ & $(85.86-108.97)$ & 0.578 & 0.093 \\
\hline$A \beta 1-42$ & $21.57 \pm 4.26$ & $(19.02-24.12)$ & $20.89 \pm 4.11$ & $(17.77-24.01)$ & 0.727 & 0.634 \\
\hline$A \beta 1-42 / A \beta 1-40$ ratio & $0.232 \pm 0.039$ & $(0.213-0.252)$ & $0.214 \pm 0.017$ & $(0.190-0.238)$ & 0.230 & 0.244 \\
\hline
\end{tabular}

Plasma $A \beta 1-40$ and $A \beta 1-42$ concentrations measured using the Amyblood test ( $A D x$ Neurosciences), and their ratio (A $\beta 1-42 / A \beta 1-40)$ were compared between cognitively normal individuals with low brain $A \beta$ load $(A \beta-)$ and high brain $A \beta$ load $(A \beta+)$ using linear models. All participants were further categorised into subjective memory complainers (SMC, $n=75)$ and non-SMC $(n=20)$. Data are presented in mean \pm SD in pg/mL. $p$ values in bold font were considered significant $(p<0.05)$.

${ }^{a} p$ represents $p$ values adjusted for age, sex, and APOE $\varepsilon 4$ status.

$\varepsilon 4$ allele status was generated, and was observed to have an area under the ROC curve (AUC (confidence interval, $\mathrm{CI})$ ) of $0.782(\mathrm{CI}=0.684-0.880)$ and was outperformed by GFAP alone (AUC $=0.795, \mathrm{CI}=0.703-0.888$, sensitivity $=$ $73 \%$, specificity $=72 \%, p($ GFAP $)=0.0001)$, base + GFAP $(\mathrm{AUC}=0.906, \mathrm{CI}=0.849-0.964$, sensitivity $=85 \%$, specificity $=80 \%, \quad p($ GFAP $)=0.00006), \quad$ base $+\mathrm{A} \beta 1-42 /$ $\mathrm{A} \beta 1-40$ ratio $(\mathrm{AUC}=0.842, \mathrm{CI}=0.758-0.926$, sensitivity $=85 \%$, specificity $=74 \%, p(\mathrm{~A} \beta 42 / \mathrm{A} \beta 40$ ratio $)=0.013)$, and base $+\mathrm{GFAP}+\mathrm{A} \beta 1-42 / \mathrm{A} \beta 1-40$ ratio $(\mathrm{AUC}=0.919$, $\mathrm{CI}=0.867-0.972, \quad$ sensitivity $=91 \%, \quad$ specificity $=80 \%$, $p(\mathrm{GFAP})=0.0001, p(\mathrm{~A} \beta 1-42 / \mathrm{A} \beta 1-40$ ratio $)=0.042)$ in distinguishing $A \beta+$ from $A \beta-$ participants (Fig. 2).

\section{Association of plasma GFAP with AD risk factors, cognitive measures, and neurodegeneration blood marker, NF-L, in all participants}

Plasma GFAP levels correlated with age $\left(r_{\mathrm{s}}=0.342, p=\right.$ $0.001)$, however, no significant association was observed with sex (mean $\pm \mathrm{SD}(\mathrm{pg} / \mathrm{mL})$ : males $=160.22 \pm 50.89$; females $=192.25 \pm 110.56 ; p=0.128)$ and $A P O E$ \&4 allele carriage $\quad($ mean $\pm \mathrm{SD} \quad(\mathrm{pg} / \mathrm{mL}):$ non-carriers $=181.98 \pm$ 102.44; $\quad$ carriers $=181.62 \pm 68.31 ; \quad p=0.988$ ). Plasma GFAP was observed to have a significant inverse correlation with working memory and executive function $\left(r_{\mathrm{s}}=\right.$ $-0.230, p=0.024)$, but not with verbal, visual, and episodic memory $\left(r_{\mathrm{s}}=-0.131, p=0.204\right)$, while an inverse trend towards statistical significance was observed for the global composite score $\left(r_{\mathrm{s}}=-0.185, p=0.072\right)$. Plasma GFAP levels were not observed to be significantly different between SMCs and non-SMCs (mean \pm SD $(\mathrm{pg} / \mathrm{mL}): \quad$ non-SMC $=167 \pm 66.71 ; \quad \mathrm{SMC}=186.34 \pm$ 103.48; $p=0.411)$. Plasma GFAP was also observed to correlate with plasma NF-L $\left(r_{\mathrm{s}}=0.441, p=7 \mathrm{E}-7\right)$.

\section{Discussion}

Findings from the current study show that plasma GFAP levels are increased in cognitively normal older adults with high brain $A \beta$ load, indicating that elevated plasma GFAP may serve as an early blood-based biomarker to identify individuals at risk of $\mathrm{AD}$, prior to the manifestation of clinical symptoms. Our observations build on previous reports of elevated plasma GFAP levels in symptomatic early-onset $\mathrm{AD}$ and late-onset $\mathrm{AD}^{7,8}$. Further, observations from our study also show that GFAP along with the common AD risk factors (age, sex, and $A P O E$ \&4 carriage), and plasma $A \beta 1-42 / A \beta 1-40$ ratio (a blood-based biomarker associated with brain $A \beta$ status ${ }^{13,15}$ ) distinguished between $A \beta$ - and $A \beta+$ individuals with $90 \%$ sensitivity and $80 \%$ specificity, wherein GFAP and plasma $A \beta 1-42 / A \beta 1-40$ ratio were statistically significant additional predictors of brain $A \beta$ load status, over and above the base model.

GFAP is one of the main intermediate filament proteins in astrocytes that has been thought to be involved in (i) fundamental cellular processes, such as cellular motility $^{26,27}$, proliferation ${ }^{28,29}$, and vesicle trafficking ${ }^{30}$, (ii) interactions between astrocytes and neurons ${ }^{31-33}$, (iii) maintenance of the integrity of the blood-brain barrier and central nervous system myelination ${ }^{34,35}$, and (iv) 
A. BM

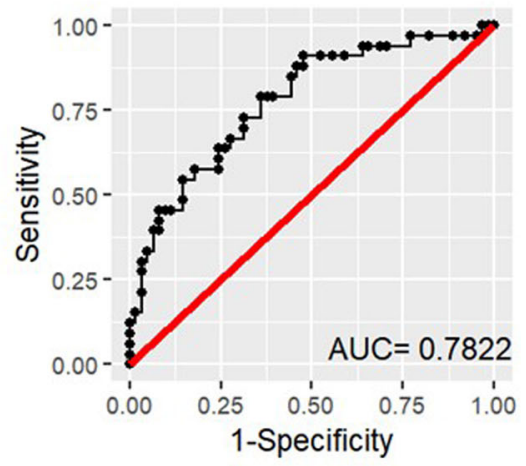

C. Plasma $A \beta 1-42 / A \beta 1-40$ ratio

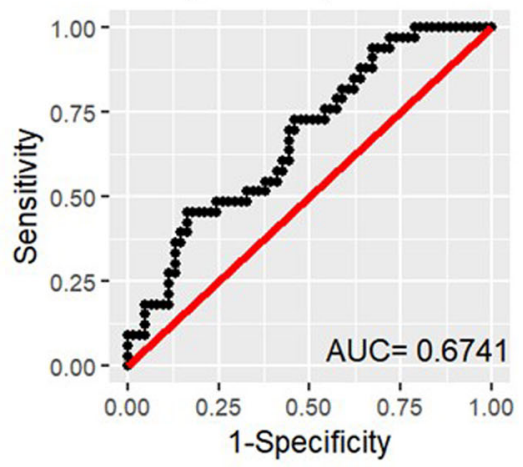

E. $\mathrm{BM}+$ plasma $\mathrm{A} \beta 1-42 / \mathrm{A} \beta 1-40$ ratio

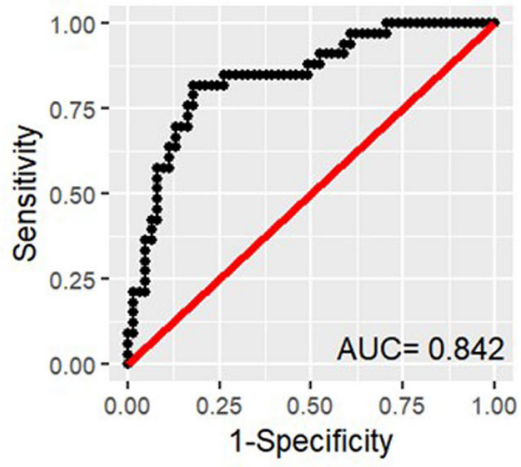

B. Plasma GFAP

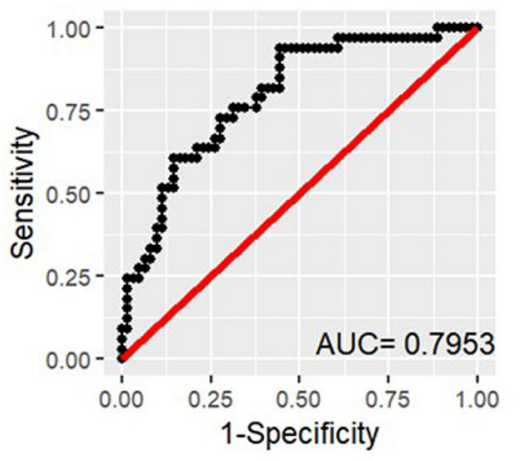

D. $\mathrm{BM}+$ plasma GFAP

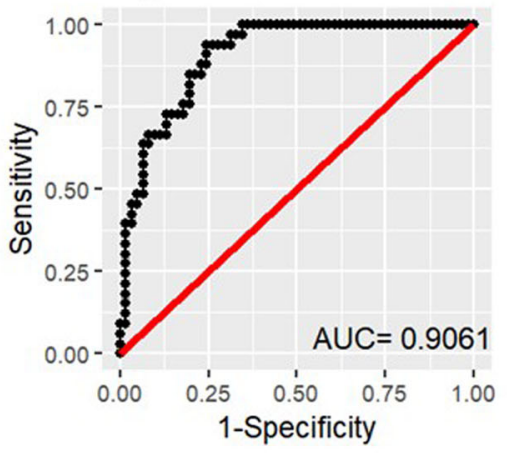

F. $\mathrm{BM}+$ plasma $\mathrm{GFAP}+\mathrm{A} \beta 1-42 / \mathrm{A} \beta 1-40$ ratio

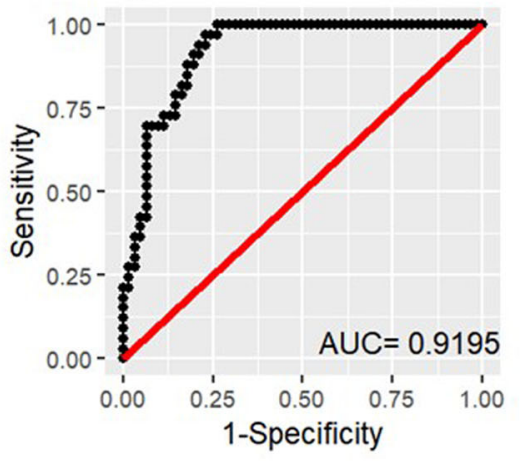

Fig. 2 Receiver operating characteristic curves for the prediction of $\mathbf{A} \boldsymbol{\beta}+$ versus $\mathbf{A} \boldsymbol{\beta}$ - participants. Receiver operating characteristic (ROC) curves are presented for $\mathbf{A}$ the 'base' model (BM) comprising major risk factors age, sex, and APOE $\varepsilon 4$ allele status $(C l=0.684-0.880)$, $\mathbf{B}$ plasma GFAP $(\mathrm{Cl}=0.703-0.888), \mathbf{C}$ plasma $\mathrm{A} \beta 1-42 / \mathrm{A} \beta 1-40$ ratio $(\mathrm{Cl}=0.563-0.785), \mathbf{D} \mathrm{BM}+\mathrm{GFAP}(\mathrm{Cl}=0.849-0.964), \mathbf{E} \mathrm{BM}+$ plasma $A \beta 1-42 / \mathrm{A} \beta 1-40$ ratio $(\mathrm{Cl}=$ 0.758-0.926), and $\mathbf{F} B M+G F A P+$ plasma $A \beta 1-42 / A \beta 1-40$ ratio $(C l=0.867-0.972)$. The base model was outperformed by models $\mathbf{B}, \mathbf{D}-\mathbf{F}$. Logistic regression models were employed to perform the analyses. Data from 94 participants were utilised for the analyses $(A \beta-, n=61 ; A \beta+, n=33)$. GFAP glial fibrillary acidic protein, AUC area under the curve, Cl confidence interval.

protection after neuronal injury ${ }^{36,37}$. It could be posited that the elevated plasma GFAP levels observed in the $\mathrm{A} \beta+$ individuals within the current study are attributed to a compromised blood-brain barrier along with an upregulation of GFAP, following astrogliosis, resulting in higher blood GFAP levels in $\mathrm{A} \beta+$ individuals. Findings from the current study are consistent with previous reports of higher GFAP expression in $A D$ brain tissue ${ }^{38}$, and the association between $A \beta$ plaques and a neuroinflammatory response, with astroglial activation and increased GFAP expression ${ }^{5,39-42}$. Furthermore, in line with our findings, PET studies using tracer ${ }^{11} \mathrm{C}$-deuterium-L-deprenyl (used for visualisation of activated astrocytes) also suggest that reactive astrocytosis is potentially a prodromal feature in $\mathrm{AD}$ development ${ }^{4,43,44}$. 
Interestingly, within the current study, plasma GFAP was observed to be significantly higher in the $A \beta+$ group (compared to the $\mathrm{A} \beta$ - group), in both SMCs and nonSMCs after adjusting for potential confounding variables, although the significance level observed in SMCs was to a much greater extent compared to that observed in the non-SMCs. However, plasma $A \beta 1-42 / A \beta 1-40$ ratios were only significantly lower in $A \beta+$ participants (compared to the $A \beta$ - participants) within the SMC subset, after stratifying participants based on their SMC status. This observation is consistent with a previous study employing the same cohort using a different $A \beta$ measurement assay ${ }^{13}$.

In addition, the current study also stratified participants based on $A P O E$ \&4 allele carriage and observed that while GFAP was significantly higher in the $A \beta+A P O E \varepsilon 4$ noncarriers (compared to the $\mathrm{A} \beta-A P O E \varepsilon 4$ non-carriers), and A $\beta 1-42 / A \beta 1-40$ ratios were significantly lower in the $A \beta+$ $A P O E$ \&4 non-carriers (compared to the $A \beta-A P O E \varepsilon 4$ non-carriers), plasma GFAP levels, and $A \beta 1-42 / A \beta 1-40$ ratios were not significantly altered in $A \beta+$ versus $A \beta$ $A P O E \& 4$ carriers. This observation could be attributed to the modest sample size of the $\varepsilon 4$ carrier subset available within the current study. Nonetheless, the observations of plasma GFAP and $A \beta 1-42 / A \beta 1-40$ ratios remaining significantly altered between $A \beta-$ and $A \beta+$ participants within the $A P O E \varepsilon 4$ non-carrier subset from this exploratory analysis may be viewed as a beneficial feature for early $\mathrm{AD}$ biomarkers, given that the presence of the $A P O E \varepsilon 4$ allele in itself is a major risk factor for the disease.

Within the current study, we observed a correlation between plasma GFAP and age, consistent with a previous report $^{8}$. Increased GFAP expression with age has also been reported in the brain ${ }^{45,46}$, caused by increased GFAP transcription, which has been suggested to be caused due to increased oxidatively damaged protein during ageing ${ }^{47}$. In addition, within the current study, plasma GFAP inversely correlated with cognitive performance, particularly with working memory and executive function; however, further studies are required to validate these observations.

Interestingly, we observed a highly significant correlation between plasma GFAP and plasma NF-L (comparison of plasma NF-L levels between $A \beta-$ and $A \beta+$ participants are presented in Supplementary Table 3). This correlation observed between plasma GFAP and plasma NF-L, revealing the association between astrocytic damage and axonal damage, is consistent with previous reports ${ }^{48,49}$.

In addition, the presence GFAP-IgG seropositivity in autoimmune GFAP astrocytopathy, an autoimmune disease of the nervous system ${ }^{50}$ and the increased risk of $\mathrm{AD}$ in individuals with autoimmune diseases ${ }^{51}$, along with our observations of increased plasma GFAP in individuals at risk of $\mathrm{AD}$, warrant further investigation into the link between $\mathrm{AD}$ and autoimmune disorders, and the investigation of possible mechanisms associated with this link.

It is acknowledged that the current study has limitations, given its modest sample size and cross-sectional design, particularly after stratifying the cohort into SMC and non-SMC subsets or APOE \&4 non-carrier and carrier subsets. Therefore, further studies are required to validate the current findings in larger independent cohorts, using both cross-sectional and longitudinal study designs. Longitudinal studies will provide more insight into the trajectory of plasma GFAP alterations associated with the progression of $\mathrm{AD}$ pathogenesis. In addition, since increased plasma GFAP has been reported to be associated with other dementias ${ }^{8}$ and neurodegenerative disorders $^{49,52}$, the specificity of GFAP as a biomarker to identify cognitively normal older adults at risk of $\mathrm{AD}$ warrants further research. However, it must also be noted that while GFAP may be associated with other dementias and neurodegenerative disorders, our data clearly show a significant positive association between GFAP and brain A $\beta$ load measured by PET (Supplementary Fig. 2), a gold standard biomarker for AD.

To conclude, the current study is the first to demonstrate increased plasma GFAP levels in cognitively normal older adults at risk of $\mathrm{AD}$. These observations suggest that astrocytic damage begins from the pre-symptomatic stage of $\mathrm{AD}$ and is associated with brain $\mathrm{A} \beta$ load. Further, observations from the current study show that the combination of plasma GFAP and plasma $A \beta 1-42 / A \beta 1-40$ ratios along with the major $\mathrm{AD}$ risk factors, have the potential to differentiate between $A \beta+$ and $A \beta$ - individuals, albeit further studies in independent cohorts are required to validate these findings. The utilisation of plasma GFAP to identify individuals at risk of $\mathrm{AD}(\mathrm{A} \beta+$ individuals), decades before the onset of $\mathrm{AD}$ clinical symptoms for clinical trials could assist with reducing the considerable screening costs, thereby facilitating much needed prevention programmes and clinical intervention trials.

\footnotetext{
Acknowledgements

We thank the participants and their families for their participation and cooperation, and Anglicare, the KaRa Institute of Neurological Diseases, and the Australian Alzheimer's Research Foundation (AARF) research and support staff for their contributions to this study. We also thank the staff of the Macquarie Medical Imaging centre in Macquarie University Hospital, Sydney, for their contributions. Florbetaben is a proprietary PET radiopharmaceutical owned by Life Molecular Imaging. For this study, Florbetaben was manufactured and supplied under GMP conditions by Cyclotek (Australia) Pty Ltd. We thank Dr. Abhay K. Singh for his help with using R. H.R.S. research is partially supported by Australian Alzheimer's Research Foundation. H.Z. is a Wallenberg Scholar supported by grants from the Swedish Research Council (\#2018-02532), the European Research Council (\#681712), Swedish State

Support for Clinical Research (\#ALFGBG-720931), the Alzheimer Drug Discovery Foundation (ADDF), USA (\#201809-2016862), and the UK Dementia Research Institute at UCL. K.B. is supported by the Swedish Research Council (\#201700915), the Alzheimer Drug Discovery Foundation (ADDF), USA (\#RDAPB201809-2016615), the Swedish Alzheimer Foundation (\#AF-742881),

Hjärnfonden, Sweden (\#FO2017-0243), the Swedish state under the agreement between the Swedish government and the County Councils, the ALF-
} 
agreement (\#ALFGBG-715986), and European Union Joint Program for Neurodegenerative Disorders (JPND2019-466-236). The Amyblood test was developed in the course of a project funded by Health Holland (PIs Teunissen and Thijssen) at Amsterdam UMC.

\section{Author details}

'Department of Biomedical Sciences, Macquarie University, North Ryde, NSW, Australia. ${ }^{2}$ School of Medical and Health Sciences, Edith Cowan University, Joondalup, WA, Australia. ${ }^{3}$ ADx NeuroSciences, Gent, Belgium. ${ }^{4}$ KaRa Institute of Neurological Diseases, Macquarie Park, NSW, Australia. ${ }^{5}$ Anglicare, Castle Hill Sydney, NSW, Australia. ${ }^{6}$ School of Psychiatry and Clinical Neurosciences, University of Western Australia, Crawley, WA, Australia. ${ }^{7}$ The Cooperative Research Centre for Mental Health, Carlton South, Australia. ${ }^{8}$ Department of Molecular Imaging \& Therapy, Austin Health, Melbourne, VIC, Australia. ${ }^{9}$ Neurochemistry Laboratory, Department of Clinical Chemistry, Amsterdam Neuroscience, Amsterdam University Medical Centers, Amsterdam, Netherlands. ${ }^{10}$ Australian Alzheimer's Research Foundation, Nedlands, WA, Australia. ${ }^{11}$ Centre for Healthy Ageing, School of Psychology and Exercise Science, College of Science, Health, Engineering and Education, Murdoch University, Murdoch, WA, Australia. ${ }^{12}$ Department of Psychiatry and Neurochemistry, Institute of Neuroscience and Physiology, University of Gothenburg, Mölndal, Sweden. ${ }^{13} \mathrm{Clinical}$ Neurochemistry Laboratory, Sahlgrenska University Hospital, Mölndal, Sweden. ${ }^{14}$ Department of Neurodegenerative Disease, UCL Institute of Neurology, Queen Square, London, United Kingdom. ${ }^{15}$ UK Dementia Research Institute at UCL, London, UK. ${ }^{16}$ Biomarkable, Gent, Belgium

\section{Author contributions}

P.C. and R.N.M. conceived the study. P.C. conducted the statistical analyses and wrote the manuscript. E.S., I.M.W.V., C.E.T., and H.V. measured plasma GFAP, $A \beta 1-40$, and $A \beta 1-42$ using the Simoa platform. H.Z. and K.B. measured plasma NF-L using the Simoa platform. All authors critically reviewed the manuscript.

\section{Conflict of interest}

E.S. is an employee of ADx NeuroSciences. HRS has received ruminations for working with Pfizer and Takeda. H.Z. has served at scientific advisory boards for Denali, Roche Diagnostics, Wave, Samumed, Siemens Healthineers, Pinteon Therapeutics, and CogRx, has given lectures in symposia sponsored by Fujirebio, Alzecure, and Biogen, and is a co-founder of Brain Biomarker Solutions in Gothenburg AB (BBS), which is a part of the GU Ventures Incubator Program (outside submitted work). K.B. has served as a consultant, at advisory boards, or at data monitoring committees for Abcam, Axon, Biogen, JOMDD/ Shimadzu. Julius Clinical, Lilly, MagQu, Novartis, Roche Diagnostics, and Siemens Healthineers, and is a co-founder of Brain Biomarker Solutions in Gothenburg AB (BBS), which is a part of the GU Ventures Incubator Program. $\mathrm{H}$. M.V. is a co-founder of ADx NeuroSciences and a founder of Biomarkable. All other authors report no conflict of interest in relation to the current study.

\section{Publisher's note}

Springer Nature remains neutral with regard to jurisdictional claims in published maps and institutional affiliations.

Supplementary Information accompanies this paper at (https://doi.org/ 10.1038/s41398-020-01137-1).

Received: 2 July 2020 Revised: 26 September 2020 Accepted: 7 October 2020

Published online: 11 January 2021

\section{References}

1. World Alzheimer Report 2019. Attitudes to Dementia (Alzheimer's Disease International, London, 2019).

2. Villemagne, V. L. et al. Amyloid beta deposition, neurodegeneration, and cognitive decline in sporadic Alzheimer's disease: a prospective cohort study. Lancet Neurol. 12, 357-367 (2013).

3. Colangelo, A. M., Alberghina, L. \& Papa, M. Astrogliosis as a therapeutic target for neurodegenerative diseases. Neurosci. Lett. 565, 59-64 (2014).
4. Carter, S. F. et al. Evidence for astrocytosis in prodromal Alzheimer disease provided by 11C-deuterium-L-deprenyl: a multitracer PET paradigm combining 11C-Pittsburgh compound B and 18F-FDG. J. Nucl. Med. 53, 37-46 (2012).

5. Muramori, F., Kobayashi, K. \& Nakamura, I. A quantitative study of neurofibrillary tangles, senile plaques and astrocytes in the hippocampal subdivisions and entorhinal cortex in Alzheimer's disease, normal controls and nonAlzheimer neuropsychiatric diseases. Psychiatry Clin. Neurosci. 52, 593-599 (1998).

6. Ishiki, A. et al. Glial fibrillar acidic protein in the cerebrospinal fluid of Alzheimer's disease, dementia with Lewy bodies, and frontotemporal lobar degeneration. J. Neurochem. 136, 258-261 (2016).

7. Elahi, F. M. et al. Plasma biomarkers of astrocytic and neuronal dysfunction in early- and late-onset Alzheimer's disease. Alzheimers Dement. 16, 681-695 (2020).

8. Oeckl, P. et al. Glial fibrillary acidic protein in serum is increased in alzheimer's disease and correlates with cognitive impairment. J. Alzheimers Dis. 67, 481-488 (2019).

9. Verberk, I. M. W. et al. Plasma Amyloid, GFAP and NfL as Predictors of Alzheimer's Pathology. (AAIC Oral Presentation, Amsterdam 2019).

10. Asih, P. R. et al. Clearing the amyloid in Alzheimer's: progress towards earlier diagnosis and effective treatments - an update for clinicians. Neurodegener. Dis. Manag. 4, 363-378 (2014).

11. Dubois, B. et al. Advancing research diagnostic criteria for Alzheimer's disease: the IWG-2 criteria. Lancet Neurol. 13, 614-629 (2014).

12. Nakamura, A. et al. High performance plasma amyloid-beta biomarkers for Alzheimer's disease. Nature 554, 249-254 (2018).

13. Chatterjee, P. et al. Ultrasensitive detection of plasma amyloid-beta as a biomarker for cognitively normal elderly individuals at risk of Alzheimer's disease. J. Alzheimers Dis. 71, 775-783 (2019).

14. Ovod, V. et al. Amyloid beta concentrations and stable isotope labeling kinetics of human plasma specific to central nervous system amyloidosis. Alzheimers Dement. 13, 841-849 (2017).

15. Doecke, J. D. et al. Total Abeta42/Abeta40 ratio in plasma predicts amyloid-PET status, independent of clinical AD diagnosis. Neurology 94, e1580-e1591 (2020).

16. Goozee, K. et al. Elevated plasma ferritin in elderly individuals with high neocortical amyloid- $\beta$ load. Mol. Psychiatry 3, 1807-1812 (2017).

17. Rossetti, H. C., Lacritz, L. H., Cullum, C. M. \& Weiner, M. F. Normative data for the Montreal Cognitive Assessment (MoCA) in a population-based sample. Neurology 77, 1272-1275 (2011).

18. McKhann, G. M. et al. The diagnosis of dementia due to Alzheimer's disease: recommendations from the National Institute on Aging-Alzheimer's Association workgroups on diagnostic guidelines for Alzheimer's disease. Alzheimers Dement. 7, 263-269 (2011).

19. Folstein, M. F., Folstein, S. E. \& McHugh, P. R. "Mini-mental state". A practical method for grading the cognitive state of patients for the clinician. J. Psychiatr. Res. 12, 189-198 (1975).

20. Chatterjee, P. et al. Association of plasma neurofilament light chain with neocortical amyloid-beta load and cognitive performance in cognitively normal elderly participants. J. Alzheimers Dis. 63, 479-487 (2018).

21. Bourgeat, P. et al. Comparison of MR-less PiB SUVR quantification methods. Neurobiol. Aging 36(Suppl 1), S159-S166 (2015).

22. Thijssen, E. et al. A Prototype Simoa Assay Quantifying Plasma Amyloid Beta 1-42 and 1-40 Isoforms can Differentiate AD from Healthy Control Subjects. (AAIC Oral Presentation, Amsterdam, 2018).

23. Bard, F. et al. Epitope and isotype specificities of antibodies to beta -amyloid peptide for protection against Alzheimer's disease-like neuropathology. Proc. Natl Acad. Sci. USA 100, 2023-2028 (2003).

24. Chatterjee, P. et al. Plasma neurofilament light chain and amyloid-beta are associated with the kynurenine pathway metabolites in preclinical Alzheimer's disease. J. Neuroinflammation 16, 186 (2019).

25. Polvikoski, T. et al. Apolipoprotein E, dementia, and cortical deposition of betaamyloid protein. N. Engl. J. Med. 333, 1242-1247. (1995).

26. Elobeid, A., Bongcam-Rudloff, E., Westermark, B. \& Nister, M. Effects of inducible glial fibrillary acidic protein on glioma cell motility and proliferation. J. Neurosci. Res. 60, 245-256 (2000).

27. Lepekhin, E. A. et al. Intermediate filaments regulate astrocyte motility. J. Neurochem. 79, 617-625 (2001).

28. Rutka, J. T. \& Smith, S. L. Transfection of human astrocytoma cells with glia fibrillary acidic protein complementary DNA: analysis of expression, proliferation, and tumorigenicity. Cancer Res. 53, 3624-3631 (1993). 
29. Toda, M. et al. Suppression of glial tumor growth by expression of glial fibrillary acidic protein. Neurochem. Res. 24, 339-343 (1999).

30. Potokar, M. et al. Cytoskeleton and vesicle mobility in astrocytes. Traffic $\mathbf{8}$, 12-20 (2007).

31. McCall, M. A. et al. Targeted deletion in astrocyte intermediate filament (Gfap) alters neuronal physiology. Proc. Natl Acad. Sci. USA 93, 6361-6366 (1996).

32. Shibuki, K. et al. Deficient cerebellar long-term depression, impaired eyeblink conditioning, and normal motor coordination in GFAP mutant mice. Neuron 16. 587-599 (1996).

33. Emirandetti, A., Graciele Zanon, R., Sabha, M. Jr \& de Oliveira, A. L. Astrocyte reactivity influences the number of presynaptic terminals apposed to spinal motoneurons after axotomy. Brain Res. 1095, 35-42 (2006).

34. Pekny, M., Stanness, K. A., Eliasson, C., Betsholtz, C. \& Janigro, D. Impaired induction of blood-brain barrier properties in aortic endothelial cells by astrocytes from GFAP-deficient mice. Glia 22, 390-400 (1998).

35. Liedtke, W. et al. GFAP is necessary for the integrity of CNS white matter architecture and long-term maintenance of myelination. Neuron 17, 607-615 (1996).

36. Tanaka, $H$. et al. Disturbance of hippocampal long-term potentiation after transient ischemia in GFAP deficient mice. J. Neurosci. Res. 67, 11-20 (2002).

37. Otani, N. et al. Enhanced hippocampal neurodegeneration after traumatic or kainate excitotoxicity in GFAP-null mice. J. Clin. Neurosci. 13, 934-938 (2006).

38. Middeldorp, J. \& Hol, E. M. GFAP in health and disease. Prog. Neurobiol. 93 421-443 (2011).

39. Simpson, J. E. et al. Astrocyte phenotype in relation to Alzheimer-type pathology in the ageing brain. Neurobiol. Aging 31, 578-590 (2010).

40. Pike, C. J., Cummings, B. J. \& Cotman, C. W. Early association of reactive astrocytes with senile plaques in Alzheimer's disease. Exp. Neurol. 132, 172-179 (1995).

41. Le Prince, G. et al. Alterations of glial fibrillary acidic protein mRNA level in the aging brain and in senile dementia of the Alzheimer type. Neurosci. Lett. 151, 71-73 (1993).
42. Li, D. et al. Neurochemical regulation of the expression and function of glial fibrillary acidic protein in astrocytes. Glia 68, 878-897 (2020).

43. Scholl, M. et al. Early astrocytosis in autosomal dominant Alzheimer's disease measured in vivo by multi-tracer positron emission tomography. Sci. Rep. 5, 16404 (2015).

44. Rodriguez-Vieitez, E. \& Nordberg, A. Imaging neuroinflammation: quantification of astrocytosis in a multitracer PET approach. Methods Mol. Biol. 1750, 231-251 (2018).

45. Goss, J. R., Finch, C. E. \& Morgan, D. G. Age-related changes in glial fibrillary acidic protein mRNA in the mouse brain. Neurobiol. Aging 12, 165-170 (1991).

46. Nichols, N. R., Day, J. R., Laping, N. J., Johnson, S. A. \& Finch, C. E. GFAP mRNA increases with age in rat and human brain. Neurobiol. Aging 14, 421-429 (1993).

47. Morgan, T. E. et al. Increased transcription of the astrocyte gene GFAP during middle-age is attenuated by food restriction: implications for the role of oxidative stress. Free Radic. Biol. Med. 23, 524-528 (1997).

48. Zeitlberger, A. M. et al. Plasma markers of neurodegeneration are raised in Friedreich's ataxia. Front. Cell Neurosci. 12, 366 (2018).

49. Abdelhak, A., Huss, A., Kassubek, J., Tumani, H. \& Otto, M. Serum GFAP as a biomarker for disease severity in multiple sclerosis. Sci. Rep. 8, 14798 (2018).

50. Fang, B. et al. Autoimmune glial fibrillary acidic protein astrocytopathy: a novel meningoencephalomyelitis. JAMA Neurol. 73, 1297-1307 (2016).

51. Wotton, C. J. \& Goldacre, M. J. Associations between specific autoimmune diseases and subsequent dementia: retrospective record-linkage cohort study, UK. J. Epidemiol. Community Health 71, 576-583 (2017).

52. Bogoslovsky, T. et al. Increases of plasma levels of glial fibrillary acidic protein, tau, and amyloid beta up to 90 days after traumatic brain injury. J. Neurotrauma 34, 66-73 (2017). 